\title{
Fluids with spin and twist
}

\author{
Larry L. Smalley \\ Department of Physics, University of Alabama, Huntsville, Alabama 35899 \\ Jean P. Krisch \\ Department of Physics, University of Michigan, Ann Arbor, Michigan 48109
}

(Received 8 August 1994; accepted for publication 19 October 1994)

\begin{abstract}
Fluids with persistent vortices that exhibit shear plus expansion (or contraction) in noninertial frames are common physical phenomena. The concept of intrinsic rotation is commonly referred to as spin; the equivalent concept for shear would be shear momenta, referred to as twist in this work. The motion of the Earth's atmosphere is a prime example of such motion in which the driving engine is the rotation of the Earth plus solar radiation. The general analytical features of persistent vortices that exhibit shear plus expansion and contraction are introduced using the methods of affine geometry. The same theoretical considerations can also be applied to astrophysical examples. (C) 1995 American Institute of Physics.
\end{abstract}

\section{INTRODUCTION}

In introducing fluids with intrinsic vorticities (spin) $)^{1-4}$ and the angular momentum associated with the shearing motion (or shear momenta, for short), we use an anology with the mechanics of elastic material subjected to strains and the consequent stresses that results. Since strains associated with shear can be thought of as a twisting of an elastic medium, we will refer to the shearing momenta throughout this work simply as twist. The concept of the mechanics of elastic media can be found in many textbooks in mechanics. ${ }^{5,6}$ In this work, we introduce a Lagrangian which is based upon elementary concepts from the theory of elasticity, and then generalize the results to affine geometry with appropriate constructs, such as frames, capable of quantifying the relationships between the stresses and the various velocity gradients exhibited by the fluid. We point out necessarily that the use of affine geometry to describe elastic and plastic deformations in matter was pioneered by Kröner. ${ }^{7-9}$ An application of a deformable, magnetically saturated, polarizable media was given by Maugin and Eringer. ${ }^{10}$ Deformations of space-time itself have been based upon the general affine group $\operatorname{GA}(4, R) .{ }^{11-16}$ Within the context of this work, Obukhov and Tresguerres have recently developed an energy-momentum tensor for a hyperfluid in metric-affine geometry. ${ }^{17}$ We will adopt the attitude that one can solve this problem in a generalized space-time and then take the appropriate limits for applications. Following the lead of Kröner, we assume the general space-time in which we are working is a metric-affine (MA) space-time. The MA approach here has commonality, in part, with the work of Obukhov and Tresguerres although this calculation follows the holonomic approach. Depending upon the limits imposed by the application, this can include both curvilinear systems as well as effective Lorentzian frames. The specialized applications (or limits) determine the arena plus the appropriate meaning of the derived quantities.

The starting point of our discussions is the velocity gradient matrix. For an isotropic fluid, the velocity gradients must not depend on the orientation of the coordinate system. The velocity gradient tensor will have the general form of a dyad in vector notation

$$
[\nabla v]_{i j}=\partial_{i} v_{j} \rightarrow \nabla_{i} v_{j}
$$

where $\nabla$ is the gradient operator and $\nabla_{i}$ is the covariant derivative which represents the generalization to affine geometry. 
In the next section, we will decompose Eq. (1) into its irreducible parts with respect to the symmetric group, introducing the symmetric, antisymmetric, and trace parts as well as the consequences suggested by this decomposition. Our approach is a self-consistent approach based upon the combination of a Lagrangian formulation plus thermodynamics. ${ }^{18-20}$ We then introduce the Lagrangian for a fluid in a general metric-affine (MA) geometry in Sec. III with appropriate thermodynamics for the fluid variables. In Sec. IV we outline the calculation using the method of independent metric and connection (or the concept of hypermomentum) and show how to convert it to the standard form of an energy-momentum tensor for a fluid. In Sec. V we repeat the calculations in Secs. III and IV for a volume conserving geometry. ${ }^{21}$ We give our conclusions and suggestions in the last section.

\section{DECOMPOSITION OF THE VELOCITY GRADIENT TENSOR}

The velocity gradient tensor can be decomposed into its symmetric and antisymmetric parts

$$
\nabla_{i} v_{j}=\nabla_{\left[i v_{j]}\right.}+\nabla_{\left(i, v_{j}\right)}
$$

The antisymmetric part can be interpreted, in the usual way, as an angular velocity corresponding to a rigid rotation of the fluid. We further interpret this as the spin (or vortex) angular velocity which further implies that the form of the spin energy density will have the value

$$
T_{S}=\frac{1}{2} \omega_{i j} S^{i j},
$$

where $S_{i j}$ is the spin density of the fluid and the spin angular velocity $\omega_{i j}$ is the logical extension of the antisymmetric part of the velocity gradient tensor

$$
\nabla_{[i} v_{j]} \rightarrow \omega_{i j} .
$$

We further adopt the model of a spin fluid described by Halbwachs ${ }^{21}$ in which the spin angular velocity is written in terms of an anholonomic frame described by the set of tetrads $a^{\alpha}{ }_{i}$ (i.e., frame) where Greek indices $\alpha=1,2,3,4$ are the anholonomic coordinates and Latin indices $i=0$, $1,2,3$ represent the holonomic coordinates such that

$$
a^{\alpha}{ }_{i} a_{\alpha j}=g_{i j}, \quad a_{k}^{\alpha} a^{\beta k}=\eta^{\alpha \beta},
$$

where $g_{i j}$ is the holonomic metric and $\eta^{\alpha \beta}$ is the anholonomic metric which in Riemannian or Riemann-Cartan geometry can be taken as an orthonormal frame. However in affine geometry, the orthogonality of the anholonomic frame may have to be relaxed, as we will show later. ${ }^{11}$ For convenience, we let the fourth component of the tetrads be the fluid four-velocity $u^{k}=a^{4 k}$.

In affine geometry, the metric is not necessarily covariantly constant, and therefore the concept of nonmetricity $Q_{i j k}$ is introduced by the definition ${ }^{22,23}$

$$
\nabla_{i} g_{j k} \equiv-Q_{i j k}
$$

From the spin analogy, ${ }^{19,21}$ we have

$$
\omega_{i j}=\dot{a}_{[i}^{\alpha} a_{\alpha j]}=\dot{a}_{[\alpha i} a^{\alpha}{ }_{j]},
$$

where the dot over the tetrad means the covariant derivative along fluid flow lines $\dot{a}^{\alpha}{ }_{i}=u^{k} \nabla_{k} a^{\alpha}{ }_{i}$, square brackets mean antisymmetrization of holonomic indices, and the last equality follows from the symmetry of the metric through $\dot{g}_{[i j]}=0$.

From the symmetric part of the (stress) velocity gradient matrix we define an equivalent twist velocity tensor 


$$
v_{i j} \equiv \frac{1}{2}\left[\dot{a}_{(i}^{\alpha} a_{\alpha j)}+\dot{a}_{(\alpha i} a^{\alpha}{ }_{j)},\right.
$$

where the parentheses indicate symmetrization of the holonomic indices. The two terms in the bracket are not identical as was in the spin angular velocity case since the holonomic metric is symmetric and $\dot{g}_{i j} \neq 0$. One can show

$$
v_{i j}=\frac{1}{2} \dot{g}_{i j}
$$

According to the Halbwachs perscription, the spin density can be written in terms of the tetrads $^{21}$ as

$$
S_{i j} \equiv \rho s_{i j}=\rho k(x)\left(a^{1}{ }_{i} a^{2}-a_{i}^{2} a^{1}{ }_{j}\right),
$$

where $\rho$ is the fluid density, $k(x)$ is the spin module function, and $s_{i j}$ is the spin per particle. In RC space-time, $k(x)$ is covariantly constant and equals the spin (per particle) in the fluid frame ${ }^{24}$ Note that the Halbwachs definition of the spin density satisfies the Frenkel condition $S_{i j} u^{j}=0 .{ }^{25}$ This further requires in this construction that the four-velocity be orthogonal to the spatial components of the tetrads.

By analogy we introduce the shear momenta density or for short, the twist density

$$
Y_{i j} \equiv \rho \tau_{i j}=\rho \tau(x)\left(a^{1}{ }_{i} a^{2}+a^{1}{ }_{i} a_{j}{ }_{j}\right)
$$

where $\tau_{i j}$ is the twist per particle and $\tau(x)$ is the twist module function. The hyperfluid approach of Obukhov and Tresguerres differs from the above assumption of separate spin and twist module functions by assuming a single specific hypermomentum density. ${ }^{17}$ By construction, $Y_{i j} u^{j}=0$. In the variational problem which we introduce, neither of the parametric functions $k(x)$ nor $\tau(x)$ are varied in the variation. The energy density for the twist/stress now becomes

$$
T_{T}=\frac{1}{2} v_{i j} Y^{i j}
$$

The introduction of the twist velocity tensor shows the importance of nonmetricity via Eq. (9). We can further decompose $v_{i j}$ into a trace

$$
v^{t}=v_{i}^{i}=\frac{1}{2} \dot{g}_{i j} g^{i j},
$$

and a trace-free part called the proper velocity gradients by subtracting the diagonal compressive (expansive) stresses given by Eq. (13)

$$
\hat{v}_{i j}=\frac{1}{2} \dot{g}_{i j}-\frac{1}{4} g_{i j} v^{t}
$$

[Note that in fluids the trace and trace-free parts may enter the description of a fluid with different couplings parameters (or strengths).] Similarly the twist can be decomposed into a trace part

$$
\tau^{t} \equiv \tau_{i}^{i}=2 \tau a^{1} a^{2 i}=2 \tau \eta^{12}
$$

and a proper twist

$$
\bar{\Upsilon}_{i j}=\rho \bar{\tau}_{i j}=\rho \tau_{i j}-\frac{1}{3} h_{i j} \rho \tau^{t}
$$

where the bar over the tensor represents the trace-free or proper twist, and the projection operator $h_{i j}=g_{i j}+u_{i} u_{j}$ insures that the proper twist satisfies the Frenkel condition. Note that the trace of the twist velocity tensor is nonzero if the Weyl vector $Q_{k} \equiv \frac{1}{4} Q_{k x}{ }^{x} \neq 0$. Thus a nonzero Weyl vector induces a nondiagonal anholonomic metric since, necessarily, $\eta^{12} \neq 0$. This assumes that we use the same coupling for the trace and trace-free parts in the definition of $\bar{\tau}_{i j}$ and $\tau^{t}$ although not for 
the coupling for $s_{i j}$ and $\tau_{i j}$ because of the different module functions. We mention that the orthogonality of the anholonomic metric would prevent the appearance of diagonal fluid stresses which could be due (perhaps) to the viscosity or viscous forces in the fluid in addition to the usual hydrostatic pressure $p$ usually assumed in perfect fluids. Thus in general the transition to a deformable or elastic medium requires a non orthogonal set of tetrads. However for those geometries in which the Weyl vector vanishes, the geometry is volume conserving, ${ }^{26-28}$ thus the anholonomic metric can be essentially orthogonalized within the context of a volume conserving variational principle. By contrast, it has been noted that a nonzero Weyl vector can lead to a space-time-dependent cosmological function that can lead to de Sitter type expansion during early cosmological times and thus can provide the gravitational echo to inflationary models. ${ }^{29,30}$

Before leaving the concept of volume conserving geometries, let us introduce the volume preserving connection ${ }^{26-28}$

$$
{ }^{\dagger} \Gamma_{i j}^{k} \equiv \Gamma_{i j}^{k}-\frac{1}{2} Q_{i} \delta_{j}^{k},
$$

where, in general, the dagger " $\dagger$ " before a symbol signifies that the use of the volume conserving connection for covariant derivatives ${ }^{\dagger} \nabla_{l}$, which has the property that the second trace of the curvature tensor vanishes, i.e.,

$$
{ }^{\dagger} V_{i j}={ }^{\dagger} R_{i j k}^{k}=2 \partial_{[i}^{\dagger} \Gamma_{j] k}^{k}=0,
$$

where ${ }^{\dagger} R_{i j k}{ }^{l}$ is the volume conserving curvature tensor. Note that we use the definitions of Schouten ${ }^{22}$ except that we use Latin indices for holonomic metric and Greek indices for the anholonomic metric.

We are now ready to introduce the Lagrangian for a perfect fluid in affine geometry in the next section.

\section{SPIN-TWIST FLUID LAGRANGIAN}

\section{A. Fluid continuity constraint}

In principle there are five different ways that one can introduce the continuity constraint on fluid low in metric-affine geometry:

$$
\begin{aligned}
& \nabla_{j}\left[\rho u^{j}\right] \equiv \stackrel{\{\}}{j}_{j}\left[\rho u^{j}\right]+\rho u^{j}\left(-2 S_{j}+2 Q_{j}\right)=0, \\
& \stackrel{*}{\nabla}_{j}\left[\rho u^{j}\right] \equiv \stackrel{\{\}}{\nabla}_{j}\left[\rho u^{j}\right]+2 \rho u^{j} Q_{j}=0,
\end{aligned}
$$

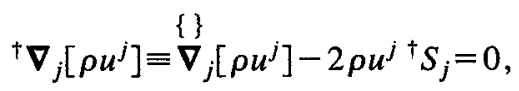

$$
\begin{aligned}
& \stackrel{*}{\nabla}\left[\rho u^{j}\right] \equiv \stackrel{\{\}}{\nabla}_{j}\left[\rho u^{j}\right]=0, \\
& e^{-1} \stackrel{*}{\nabla}_{j}\left[e \rho u^{j}\right] \equiv \stackrel{\{\}}{\nabla}_{j}\left[\rho u^{j}\right]=0,
\end{aligned}
$$

where $S_{j}=S_{j x}{ }^{x}$ is the torsion vector, and the torsion is defined as $S_{i j}{ }^{k}=\Gamma_{[i j]^{k}}$. In RiemannCartan space-time, only the constraint in the second equation yields field equations avoiding an interpretation of mass (or particle number) nonconservation as is the case with the first equation. ${ }^{19}$ In metric-affine geometry, only the last two forms of the constraint lead to mass conservation. ${ }^{26}$ However, the geometry is volume nonconserving for the fifth constraint. It is only through the field equations from the variation of the connection that eventually the Weyl vector is set to zero thereby forcing the geometry to be volume conserving! Note that either an active torsion vector or 
a Weyl vector will lead to an interpretation of particle number nonconservation. This is why the constraint given by Eq. (20) leads to such an interpretation in MA geometry. ${ }^{31}$

In this work, we will only consider the two cases given by Eqs. (19) and (22) since they are representive of the two extremes described above. In the first case, the geometry is volume nonconserving and the torsion vector is active. For the second, the geometry is volume conserving, and there is particle number conservation.

\section{B. Lagrangian for spinning fluids with twist}

Our Lagrangian will be of the form

$$
\mathscr{L}=\mathscr{L}_{\mathrm{REH}}+\mathscr{L}_{\mathrm{ST}}
$$

where the Ray-Einstein-Hilbert Lagrangian $\mathscr{L}_{\mathrm{REH}}$ is given by a geometric part

$$
\mathscr{L}_{G}=\mathscr{B} / 2 \kappa,
$$

where $\mathscr{R}$ is the scalar curvature density, $e=\sqrt{-\left|g_{i j}\right|}, \kappa=8 \pi G$, and $G$ is the gravitational constant plus a perfect fluid Lagrangian ${ }^{18}$

$$
\mathscr{B}_{F}=e\left\{-\rho\left[1+\epsilon\left(\rho, s, s_{i j}, \tau_{i j}\right)\right]+\lambda_{1}\left(u_{i} u^{i}+1\right)+\lambda_{2} \nabla_{i}\left[\rho u^{i}\right]+\lambda_{3} u^{i} X_{, i}+\lambda_{4} u^{i} s_{, i}\right\},
$$

where $\epsilon$ is the fluid energy density which is assumed to be a function of the fluid density, spin, ${ }^{19}$ entropy $s,{ }^{18}$ and the twist; ${ }^{32}$ and $\lambda_{1}$ is the Lagrange multiplier that insures orthogonality of the four-velocity, $\lambda_{2}$ is the Lagrange multiplier that insures the continuity constraint given by Eq. (18), and $\lambda_{3}$ and $\lambda_{4}$ insure that along fluid flow lines, the fluid is not irrotational through conservation the Lin function $X^{18}$ and conservation of the entropy, respectively. The spin-twist Lagrangian is given by

$$
\begin{aligned}
\mathscr{L}_{\mathrm{ST}}= & e\left\{-\frac{1}{2} \rho k\left[a^{1 i} \dot{a}^{2}{ }_{i}-\dot{a}^{1}{ }_{i} a^{2 i}\right]-\frac{1}{2} \rho \tau a^{1 i} a^{2 j} u^{k} \nabla_{k} g_{i j}+\lambda_{11}\left(a^{1}{ }_{i} a^{1 i}-\eta^{11}\right)+\lambda_{12}\left(a^{1}{ }_{i} a^{2 i}-\eta^{12}\right)\right. \\
& \left.+\lambda_{22}\left(a^{2}{ }_{i} a^{2 i}-\eta^{22}\right)+\lambda_{41} u^{i} a^{1}{ }_{i}+\lambda_{42} u^{i} a^{2}{ }_{i}\right\},
\end{aligned}
$$

where the first two terms represent the energy density of the spin and twist, respectively, written in terms of the tetrads, and the various Lagrange multipliers $\lambda_{\mu \nu}$ define the tetrads. Without loss of generality, the third component of the tetrads does not enter into the Lagrangian, by construction, when we use the Halbwachs' ansatz. ${ }^{21}$ We can further assume that the tetrads are normalizeable but not necessarily orthogonal; in particular, $\eta^{12} \neq 0$. However, by construction, we have assumed that the four-velocity is orthogonal to the spatial tetrads. ${ }^{18}$

In order to complete the self-consistent framework, we need to add the thermodynamics which takes the form

$$
d \epsilon=T d s+\frac{p}{\rho^{2}} d \rho+\frac{1}{2} \omega_{i j} d s^{i j}+\frac{1}{2} v_{i j} d \tau^{i j},
$$

since we have assumed that the energy of the fluid is a function of the spin and the twist.

The variables of the variation are then $g_{i j}, \Gamma_{k j}{ }^{l}, \rho, u^{i}, a^{1 i}, a^{2 i}, X, s$ plus the various Lagrange multipliers. An alternate independent set of geometric variables can be used by varying the metric $g_{i j}$, the nonmetricity $Q_{i j k}$, and the torsion $S_{i j}{ }^{k}$ instead of the metric $g_{i j}$ and connection $\Gamma_{k j}{ }^{l}$. The relationship between these alternate methods is seen from the variation of the gravitational Lagrangian Eq. (24) for the former set,

$$
\delta\left\{\frac{\mathscr{B}}{2 \kappa}\right\} \equiv e\left\{\frac{1}{\kappa} P_{l}^{j k} \delta \Gamma_{k j}^{l}-\frac{1}{2 \kappa} G^{(i j)} \delta g_{i j}\right\},
$$


which defines the Palatini tensor through the variation of the scalar curvature density $\mathbb{B}^{11,26-28}$

$$
P_{l}{ }^{j k} \equiv \frac{1}{2 e} \frac{\delta \mathscr{R}(g, \Gamma)}{\delta \Gamma_{k j}{ }^{l}},
$$

which then becomes

$$
P_{i j}^{k}=T_{i j}^{k}-\frac{1}{2}\left[Q_{i j}^{k}+\delta_{[i}^{k} Q_{j] x}{ }^{x}-\delta_{i}^{k} Q^{x}{ }_{j x}\right]
$$

where the modified torsion is given by

$$
T_{i j}^{k}=S_{i j}^{k}+2 \delta_{[j}^{k} S_{i] x}^{x} .
$$

Note the appearances of both traces of nonmetricity in Palatini tensor given in Eq. (31). The variation of the matter Lagrangian with respect to the connection defines the fluid hypermomentum

$$
\Delta_{l}^{j k} \equiv-\frac{1}{e} \frac{\delta \mathscr{L}_{\text {matter }}}{\delta \Gamma_{k j}^{l}}
$$

so that the hypermomentum field equation becomes

$$
P_{l}^{j k}=\kappa \Delta_{l}^{j k}
$$

Also for the $\Gamma_{k j}^{l}$ variation, the symmetric components of the Einstein tensor are defined as

$$
G^{(i j)} \equiv-\frac{1}{e} \frac{\delta \mathscr{R}}{\delta g_{i j}},
$$

so that with the $\Gamma$-metric symmetric energy-momentum tensor defined by

$$
\Gamma_{\sigma^{i j}} \equiv \frac{2}{e} \frac{\delta \mathscr{E}_{\text {matter }}}{\delta g_{i j}}
$$

one obtains the metric field equation

$$
G^{(i j)}=\kappa^{\Gamma} \sigma^{i j}
$$

An identity of the Riemann tensor in MA gives the antisymmetric part of the Einstein tensor ${ }^{22}$

$$
G^{[i j]}=e^{-1} g^{l[i} g^{j] m}{ }^{*} \nabla_{k}\left\{e\left(T_{l m}^{k}-\frac{1}{2} \delta_{[l}^{k} Q_{m] x}{ }^{x}\right)\right\}
$$

After adding Eq. (37) to Eq. (38), the right-hand side of the equation (divided by $\kappa$ ) becomes the canonical energy momentum tensor

$$
\Sigma^{i j}=\Gamma^{i j}+(\kappa e)^{-1} g^{l[i} g^{j] m} \nabla_{k}^{*}\left\{e\left(T_{l m}^{k}-\frac{1}{2} \delta_{[l}^{k} Q_{m] x}{ }^{x}\right)\right\}
$$

Note that the raising and lowering of indices is cumbersome because of the nonmetricity.

The MA variation of $\mathscr{L}_{G}$ with respect to the other independent set is given by ${ }^{33}$ 


$$
\begin{aligned}
\delta \mathscr{C}_{G}= & \frac{1}{2 \kappa}\left\{\left[-e G^{(i j)}-\stackrel{*}{\nabla}_{k}\left(e\left(P^{i(j k)}+P^{j(k i)}-P^{k(i j)}\right)\right)\right] \delta g_{i j}-2 e g_{k l}\left(P^{[i i] j}-P^{[i j] l}+P^{[j l] i}\right) \delta S_{i j}{ }^{k}\right. \\
& \left.+e\left[P^{k(j i)}+P^{j(i k)}-P^{i(k j)}\right] \delta Q_{i j k}\right\}
\end{aligned}
$$

and the improved symmetric energy-momentum defined by ${ }^{19,34}$

$$
T^{i j} \equiv \frac{2}{e} \frac{\delta \mathscr{L}_{\mathrm{matter}}}{\delta g_{i j}}
$$

so that the variation with respect to the metric of the field and the matter Lagrangians has the metric field equation with the usual meaning

$$
G^{(i j)}+e^{-1} \stackrel{*}{\nabla_{k}}\left(e\left(P^{i(j k)}+P^{j(k i)}-P^{k(i j)}\right)\right)=\kappa T^{i j} .
$$

In what follows, it is actually easier to do the variation treating the metric and connection as independent and then convert later to the metric field equation for independent metric, torsion, and nonmetricity by using the above relationships. The advantage of this approach is that the very tedious torsion and nonmetricity field equations are easily found from the variation of an independent connection.

Finally the variation using the continuity constraint given by Eq. (22) is obtained by substituting the appropriate constraint for the $\lambda_{2}$ term.

In next section, we calculate the variational equations for the continuity constraint $\nabla_{i}\left[\rho u^{i}\right]=0$.

\section{FLUID FIELD EQUATIONS WITH NONCONSERVATION OF BOTH PARTICLE NUMBER AND VOLUME}

\section{A. Variational equations for case $\nabla_{i}\left[\rho u^{\prime}\right]=0$}

Using the constraint given by Eq. (19) will yield field equations in which the torsion vector and the Weyl vector are active. Thus the anholonomic metric will in general be nonorthogonal. In addition, we will find that the Weyl vector, torsion vector, plus the other trace of the nonmetricity $Q^{x}{ }_{x j}$ cannot be resolved individually by the field equations. This is a direct result of the fact that the variational principle for an independent metric and connection given by the Lagrangian Eq. (24) with the scalar curvature Lagrangian Eq. (25) cannot resolve four of the total possible 64 degrees of freedom of the connection in the variation described by the variables $\left\{g_{i j}, \Gamma_{i j}{ }^{k}{ }^{28}\right.$ Nevertheless certain combinations of these vectors can be resolved which allows simplification of the torsion and nonmetricity field equations. As a result, this gives rise to an interesting result for the trace part of the twist. Thus it will appear, in principle, that the disadvantage of using a volume nonconserving connection can be overcome.

\section{B. Connection field equation}

The variation of Eq. (24) with respect to the connection gives the field equation for the connection: Connection $\delta \Gamma_{k j}^{\prime}$ :

$$
\lambda_{2} \rho u^{j} \delta_{l}^{k}+\frac{1}{2} u^{k} S^{j}{ }+\frac{1}{2} u^{k} \Upsilon^{j}{ }_{l}=-\frac{1}{\kappa} P_{l}^{j k}=-\Delta_{l}^{j k}
$$

From Eq. (31) for $P_{l}^{j k}$ we can resolve the four degrees of freedom discussed above by taking the trace on $j l$ since the Palatini tensor satisfies the identity $P_{l}{ }^{l k}=0$. This gives the restriction on the matter fields 


$$
P_{l}^{l k}=\kappa \lambda_{2} \rho u^{k}+(\kappa / 2) u^{k} Y_{x}^{x}=0
$$

or

$$
\lambda_{2}=-\tau \eta^{12}
$$

where we have used $Y_{x}^{x}=2 \rho \tau \eta^{12}$ from the trace of Eq. (11). From the two other traces of the Palatini tensor, one gets

$$
P_{l j}^{l}=2 S_{j}-3 Q_{j}+\frac{3}{2} Q_{x j}^{x}=-4 \kappa \lambda_{2} \rho u_{j}
$$

and

$$
P_{j l}^{l}=-2 S_{j}+Q_{j}+\frac{1}{2} Q_{x j}^{x}=-\kappa \lambda_{2} \rho u_{j}
$$

which shows that we cannot uniquely resolve the three traces of the torsion and nonmetricity from these equations. However, one interesting result that can be found is

$$
\bar{Q}_{x j}^{x}=-\frac{5}{2} \kappa \lambda_{2} \rho u_{j},
$$

where the nonmetricity which is trace-free of the Weyl vector is given by

$$
\vec{Q}_{k j l}=Q_{k j l}-Q_{k} g_{j l}
$$

However, since $Q_{l j}{ }^{k}$ has two traces, we can extract them in the form ${ }^{35}$

$$
Q_{l j}{ }^{k}=\overline{\bar{Q}}_{l j}{ }^{k}+\frac{10}{9}\left(Q_{l} \delta_{j}^{k}-\frac{1}{5} Q_{j} \delta_{l}^{k}-\frac{1}{5} Q^{k} g_{j l}\right)+\frac{2}{9}\left(Q^{x}{ }_{x j} \delta_{l}^{k}+Q_{x}{ }^{x k} g_{j l}-\frac{1}{2} Q^{x}{ }_{x l} \delta_{j}^{k}\right),
$$

where $\overline{\bar{Q}}_{l j}{ }^{k}$ is the double trace-free nonmetricity. The connection to $\bar{Q}_{l j}{ }^{k}$ is then given by

$$
\bar{Q}_{l j}{ }^{k}=\overline{\bar{Q}}_{l j}{ }^{k}+\frac{2}{9}\left(\bar{Q}_{x j}^{x} \delta_{l}^{k}+\bar{Q}_{x}^{x k} g_{j l}-\frac{1}{2} \bar{Q}_{x l}^{x} \delta_{j}^{k}\right) .
$$

From the antisymmetric components of the Palatini tensor $P_{[l j]}{ }^{k}$ and the connection field equation (43), one obtains the torsion field equation

$$
\bar{S}_{l j}^{k}-\frac{\kappa}{2} \overline{\bar{Q}}_{[l j]}^{k}=\frac{1}{2} S_{l j} u^{k}
$$

where $\bar{S}_{l j}{ }^{k}$ is the trace-free torsion. From the symmetric components of the Palatini tensor $P_{(l j)}{ }^{k}$ and Eq. (43), one obtains the nonmetricity field equation

$$
\overline{\bar{Q}}_{(l j)}{ }^{k}=-\frac{1}{9} \kappa \rho \lambda_{2}\left(2 u_{(l} \delta_{j)}^{k}+u^{k} g_{j l}+6 u^{k} u_{j} u_{l}\right)+\kappa \bar{\Upsilon}_{j l} u^{k},
$$

where the term inside the parentheses on the right-hand side insures that the right-hand side vanishes for all the traces on $\overline{\bar{Q}}_{(l j)}{ }^{k}$. Indeed these terms are the results of the nonzero trace for the twist in Eq. (44) and as a result, the nondiagonal anholonomic metric seen in Eq. (45).

\section{Metric plus fluid field equations}

The remainder of the yariational equations become Metric equation $\delta g_{i j}$ : 


$$
\begin{aligned}
& -\frac{G^{(i j)}}{2 \kappa}-\frac{1}{2} \rho(1+\epsilon) g^{i j}+\lambda_{1} u^{i} u^{j}+\lambda_{11} a^{1 i} a^{1 j}+\lambda_{22} a^{2 i} a^{2 j}+2 \lambda_{12} a^{(1 i} a^{2 j)}+2 \lambda_{41} u^{(i} a^{1 j)} \\
& +2 \lambda_{42} u^{(i} a^{2 j)}-\frac{1}{2} T_{S} g^{i j}-\frac{1}{2} T_{T} g^{i j}+\frac{1}{2} \stackrel{\nabla}{k}_{k}^{*}\left[\rho k u^{k} a^{(1 j}\right] a^{2 i)}-\rho k a^{(1 j} a^{2 i)} u^{l} Q_{l} \\
& -\frac{1}{2} \stackrel{*}{\nabla}_{k}\left[\rho k u^{k} a^{(2 j}\right] a^{(i)}+\rho k a^{(1 j} a^{2 i)} u^{l} Q_{l}+\frac{1}{2} \stackrel{*}{\nabla}_{k}\left[\rho \tau u^{k} a^{(1 j} a^{2 i)}\right]-\rho \tau a^{(1 j} a^{2 i)} u^{l} Q_{l}=0 .
\end{aligned}
$$

Fluid density $\delta \rho$ :

$$
-(1+\epsilon)-\frac{p}{\rho}-{ }^{\star} \lambda_{2}+2 \lambda_{2} u^{k} Q_{k}-\frac{1}{\rho} T_{S}-\frac{1}{\rho} T_{T}=0,
$$

where the "star" in place of the "dot" signifies $\stackrel{*}{\lambda_{2}}=u^{k} \nabla_{k}^{*} \lambda_{2}$.

Four velocity $\delta u^{k}$ :

$$
\begin{aligned}
& -\rho \stackrel{*}{\nabla}_{k} \lambda_{2}+2 \rho \lambda_{2} Q_{k}+2 \lambda_{2} U_{k}+\lambda_{3} \partial_{k} X+\lambda_{4} \partial_{k} s \\
& \quad=2 \lambda_{41} a_{k}{ }_{k}+2 \lambda_{42} a^{2}{ }_{k}-\frac{1}{2} \rho k a^{1 i} \nabla_{k} a^{2}{ }_{i}+\frac{1}{2} \rho k a^{2 i} \nabla_{k} a_{i}^{1}-\frac{1}{2} \rho \tau\left[\nabla_{k} g_{i j}\right] a^{1 i} a^{2 j}=0 .
\end{aligned}
$$

Entropy \&s:

$$
\rho T-\stackrel{*}{\nabla}_{i}\left[\lambda_{4} u^{i}\right]+2 \lambda_{4} u^{k} Q_{k}
$$

Lin constraint $\delta X$ :

$$
-\stackrel{*}{\nabla}_{i}\left[\lambda_{3} u^{i}\right]+2 \lambda_{3} u^{k} Q_{k}=0
$$

The tetrad variations

$\delta a^{1 k}$ :

$$
\begin{aligned}
& -\rho k \omega_{k i} a^{2 i}-\rho \sigma v_{i k} a^{2 i}+2 \lambda_{11} a_{k}{ }_{k}+2 \lambda_{12} a^{2}{ }_{k}+2 \lambda_{41} u_{k}-\frac{1}{2} \rho k \dot{a}_{k}{ }_{k}-\frac{1}{2} \nabla_{l}\left[\rho k a^{2 j} u^{l}\right] g_{j k} \\
& \quad+\rho k a^{2 j} u^{l} Q_{l} g_{j k}-\frac{1}{2} \rho \tau u^{l}\left[\nabla_{l} g_{j k}\right] a^{2 j}=0
\end{aligned}
$$

and $\delta a^{2 k}$ :

$$
\begin{array}{r}
\rho k \omega_{k i} a^{1 i}-\rho \sigma v_{i k} a^{1 i}+2 \lambda_{22} a_{k}^{2}+2 \lambda_{12} a_{k}^{l}+2 \lambda_{42} u_{k}-\frac{1}{2} \rho k \dot{a}_{k}{ }_{k} \\
+\frac{1}{2}{ }^{*}\left[\rho k a^{1 j} u^{l}\right] g_{j k}-\rho k a^{1 j} u^{l} Q_{l} g_{j k}-\frac{1}{2} \rho \tau u^{l}\left[\nabla_{l} g_{j k}\right] a^{1 j}=0
\end{array}
$$

The various constraint equations then completes the set of variational equations for the case with fluid continuity $\nabla_{i}\left[\rho u^{i}\right]=0$. In obtaining the above variational equations, we have ignored surface terms that arise from the variation of the action. We have explicitly used the MA identity for the covariant derivative of the determinant of the metric 


$$
\boldsymbol{\nabla}_{i} e=-2 e Q_{i}
$$

Then the formation of surface terms can be facilitated by using the results

$$
\partial_{i} \mathscr{Z}^{i}=\stackrel{\boldsymbol{\nabla}}{i}_{i}\left[e V^{i}\right]
$$

where the vector density is given by $\mathscr{T}^{i} \equiv e V^{i}$.

In the next two subsections, we resolve the above variational equations into a spin-twist transport equation plus a metric field equation by eliminating the Lagrange multipliers and specific reference to the spatial tetrads.

\section{Spin-twist transport equation}

Because Eq. (61) holds in MA geometry, certain groups of terms often occur that can be combined for convenience such as in the tetrad variation given by Eq. (60)

$$
\frac{1}{2} \nabla_{l}^{*}\left[\rho k a^{1 j} u^{l}\right] g_{j k}-\rho k a^{1 j} u^{l} Q_{l} g_{j k}=\frac{1}{2 e} \nabla_{l}^{*}\left[e \rho k a^{1 j} u^{l}\right] g_{j k}
$$

Introducing this combination of terms into the variations mimics the form of the variations using the volume preserving connection ${ }^{\dagger} \Gamma_{k j}{ }^{l}$ (except for the appearance of $e$ and the "star" derivative depending on the choice of fluid continuity constraint).

Multiplying the $\delta \rho$ variation by $\rho$, we obtain

$$
(\rho / e)\left(e \lambda_{2}\right)^{\star}=[\rho(1+\epsilon)+p]+T_{S}+T_{T},
$$

where we have used Eqs. (3) and (12). From $u^{k}$ times $\delta u^{k}$

$$
\lambda_{1}=\frac{1}{2}[\rho(1+\epsilon)+p] .
$$

From $u^{k}$ times $\delta a^{1 k}$

$$
2 \lambda_{41}=\rho k \dot{u}_{j} a^{2 j}+\rho k \dot{u}^{k} a_{k}^{2}-\rho \tau u^{k} a^{2 i} \dot{g}_{i k}
$$

From $u^{k}$ times $\delta a^{2 k}$

$$
2 \lambda_{42}=-\rho k \dot{u}_{j} a^{1 j}-\rho k \dot{u}^{k} a^{1}{ }_{k}-\rho \tau u^{k} a^{1 i} \dot{g}_{i k} .
$$

Then using the definitions of spin density Eq. (10) and twist density Eq. (11), we can find the symmetric combination of Eqs. (66) and (67)

$$
2 \lambda_{41} u^{(i} a^{1 j)}+2 \lambda_{42} u^{(i} a^{2 j)}=u^{(i} S^{j)}{ }_{l} \dot{u}^{l}+u^{(i} S^{j) l} \dot{u}_{l}-u^{(i} \Upsilon^{j) l} \dot{u}_{l}+u^{(i} \Upsilon^{j)}{ }_{l} \dot{u}^{l}
$$

and the antisymmetric combination

$$
2 \lambda_{41} u^{[i} a^{1 j]}+2 \lambda_{42} u^{[i} a^{2 j]}=u^{[i} S^{j]}{ }_{l} \dot{u}^{l}+u^{[i} S^{j] l} \dot{u}_{l}-u^{[i} \Upsilon^{j] l} \dot{u}_{l}+u^{[i} \Upsilon^{j]}{ }_{l} \dot{u}^{l}
$$

We have assumed that the anholonomic metric can be normalized; wc further assume that $\eta^{12}=a=$ const which only requires that $0 \leqslant a^{2}<1$. With this restriction, one arrives at the four equations from $a^{1 k}$ times $\delta a^{1 k}, a^{2 k}$ times $\delta a^{2 k}, a^{2 k}$ times $\delta a^{1 k}$, and $a^{1 k}$ times $\delta a^{2 k}$, respectively,

$$
\begin{aligned}
& 2 \lambda_{11}+2 \lambda_{12} \eta^{12}-2 T_{S}-2 T_{T}-\frac{1}{2} \rho \eta^{12}\left[e^{-1}(e k)^{\star}\right]=0, \\
& 2 \lambda_{22}+2 \lambda_{12} \eta^{12}-2 T_{S}-2 T_{T}+\frac{1}{2} \rho \eta^{12}\left[e^{-1}(e k)^{\star}\right]=0,
\end{aligned}
$$




$$
\begin{aligned}
& 2 \lambda_{11} \eta^{12}+2 \lambda_{12}+2 \rho \tau a_{k}^{2} \dot{a}^{2 k}-\frac{1}{2} \rho\left[e^{-1}(e k)^{\star}\right]=0, \\
& 2 \lambda_{22} \eta^{12}+2 \lambda_{12}+2 \rho \tau a_{k}^{1} \dot{a}^{1 k}+\frac{1}{2} \rho\left[e^{-1}(e k)^{\star}\right]=0 .
\end{aligned}
$$

Equations (70)-(73) imply (amongst other things) that $\lambda_{11} \neq \lambda_{22}$ and that $(e k)^{\star} \neq 0$. We will see later in the discussion of the volume conserving connection that $\eta^{12}=0$ which in turn implies that $\lambda_{11}=\lambda_{22}$, but $(e k)^{\star} \neq 0$ (unless $\tau=0$ as well). However in the analysis that we are doing here, we will not need the explicit values of these functions to continue.

The tetrad variations yield two additional relationships which we will use. The first is from the sum of the antisymmetric combinations of $a^{1 j}$ times the $\delta a^{1 k}$ variation and $a^{2 j}$ times the $\delta a^{2 k}$ variation. The similar symmetric combination will be used in the metric field equation.

For the antisymmetric combination, one gets after using Eq. (69),

$$
\begin{gathered}
\rho k \omega_{i}^{[k} s^{j] i}-2 \rho v_{i}{ }^{[k} \sigma^{j] k}-u^{[j} S^{k]}{ }_{l} \dot{u}^{l}-u^{[j} S^{k] l} \dot{u}_{l}-u^{[k} Y^{j] l} \dot{u}_{l}+u^{[k} Y^{j]}{ }_{l} \dot{u}^{l}+\frac{1}{2} \rho k\left(\dot{a}^{1}{ }_{l} g^{l[k} a^{2 j]}\right. \\
\left.-\dot{a}^{2}{ }_{l} g^{l[k} a^{1 j]}\right)+\frac{1}{2} \rho k\left(\dot{a}^{[1 k} a^{2 j]}-\dot{a}^{[2 k} a^{l j]}\right)+e^{-1} \rho(e k)^{\star} a^{[l k} a^{2 j]}=0 .
\end{gathered}
$$

One can show the following two results directly from the derivative along fluid flow lines of the definition of the spin given in Eq. (10) for the case $\nabla_{i}\left[\rho u^{i}\right]=0$ :

$$
\frac{1}{2} e \rho k\left(\dot{a}^{[1 k} a^{2 j]}-\dot{a}^{[2 k} a^{1 j]}\right)=\frac{1}{4} \rho\left(e s^{k j}\right)^{\star}-\frac{1}{2} \rho(e k)^{\star} a^{[1 k} a^{2 j]},
$$

and

$$
\frac{1}{2} \rho k\left(\dot{a}_{l}{ }_{l}{ }^{l[k} a^{2 j]}-\dot{a}^{2} g^{l[k} a^{1 j]}\right)=\frac{1}{2} \rho k\left(\dot{a}^{[1 k} a^{2 j]}-\dot{a}^{[2 k} a^{1 j]}\right)+\frac{1}{2} \rho s_{l}{ }^{[k} \dot{g}^{j l l} .
$$

Using Eq. (75), we can rewrite Eq. (76) in the form

$$
\frac{1}{2} \rho k\left(\dot{a}^{1}{ }_{l} g^{l[k} a^{2 j l}-\dot{a}^{2}{ }_{l} g^{l[k} a^{1 j]}\right)=\frac{1}{2 e} \rho\left\{\frac{1}{2}\left[e s^{j k}\right]^{\star}-\left[e s_{l}^{[k}\right]^{\star} g^{j l l}-(e k)^{\star} a^{[l k} a^{2 j]}\right\} .
$$

Then using Eqs. (75) and (77), we can rewrite Eq. (74) as

$$
\frac{\rho}{2 e}\left[e s_{l}^{[j}\right]^{\star} g^{k] l}+\rho k \omega_{i}^{[k} s^{j] i}-2 \rho v_{i}^{[k} \tau^{j] k}=u^{[j} S^{k]}{ }_{l} \dot{u}^{l}+u^{[j} S^{k] l} \dot{u}_{l}+u^{[j} Y^{k] l} \dot{u}_{l}-u^{[j} \Upsilon^{k]}{ }_{l} \dot{u}^{l}
$$

Recalling that $v^{i j}=-\frac{1}{2} \dot{g}^{i j}$ and that $h^{i j}=g^{i j}+u^{i} u^{j}$, we write Eq. (78) in the final form

$$
\frac{\rho}{e}\left[e s^{k j}\right]^{\star}-\frac{\rho}{2 e}\left[e s_{l}^{[j}\right]^{\star} g^{k] l}=\left(\dot{h}_{l}^{[j}+\frac{1}{2} \omega_{l}^{[j}\right) S^{k] l}+\left(\dot{h}^{l[j}+\frac{1}{2} \omega^{l[j}\right) S^{k]}{ }_{l}-\dot{h}_{l}^{[j} \Upsilon^{k] l}+\dot{h}^{l[j} \Upsilon^{k]}{ }_{l}
$$

Equation (79) is the MA extension of Fermi-Walker transport to a fluid with both spin $S_{i}{ }^{j}$ and twist $Y_{i}{ }^{j}$. Equation (79) also reduces to the usual Fermi-Walker transport in RC geometry ${ }^{19}$ in which the twist $Y_{i}^{j}$ vanishes and the simple relationship $2 \omega_{i}{ }^{[k} s^{j 1 i}=\dot{s}^{k j}$ holds.

In the next subsection, we develop a symmetric combination of tetrad variational equations for use in the metric field equation similar to the combination that led in the above discussion to the extended Fermi-Walker transport equation.

\section{E. Metric field equation}

The tetrad variations also yield the symmetric combination $a^{1 j}$ times the $\delta a^{1 k}$ variation plus $a^{2 j}$ times the $\delta a^{2 k}$ variation [similar to the manipulations that led to Eq. (74)]: 


$$
\begin{gathered}
\lambda_{11} a^{1 j} a^{1 i}+\lambda_{22} a^{2 j} a^{2 i}+2 \lambda_{12} a^{(1 i} a^{2 j)}+2 \lambda_{41} a^{(1 i} u^{j)}+2 \lambda_{42} a^{(2 i} u^{j)}=-\frac{1}{2} \rho \omega_{k}{ }^{(i} s^{j) k}+\rho v_{k}{ }^{(i} \tau^{j) k} \\
-\frac{1}{2} \rho k\left[\dot{a}^{(1 i} a^{2 j)}-\dot{a}^{(2 i} a^{1 j)}\right]-\frac{1}{4} \rho \dot{s}_{l}^{(i} g^{j) l}+\frac{1}{2}\left[u^{(j} S^{i)} \dot{u}^{l}+u^{(j} S^{i) l} \dot{u}_{l}-u^{(j} Y^{i) l} \dot{u}_{l}+u^{(j} Y^{i)}{ }^{l} \dot{u}^{l}\right]
\end{gathered}
$$

Upon substituting Eq. (80) along with Eqs. (64) and (65) into the metric equation (54), and rearranging, we find the symmetric components of the Einstein tensor,

$$
\begin{aligned}
G^{(i j)}= & \kappa\left\{\left([\rho(1+\epsilon)+p] u^{i} u^{j}+p g^{i j}\right)+u^{(j} S^{i)}{ }_{l} \dot{u}^{l}+u^{(j} S^{i) l} \dot{u}_{l}-u^{(j} \Upsilon^{i) l} \dot{u}_{l}+u^{(j} Y^{i)}{ }_{l} \dot{u}^{l}-\rho \omega_{k}{ }^{(i} s^{j) k}\right. \\
& \left.+2 \rho v_{k}{ }^{(i} \tau^{j) k}+\frac{1}{2} \rho \dot{s}_{l}{ }_{l}^{i} g^{j) l}+\left(\frac{1}{2 e}{ }^{*}{ }_{k}\left[e \Upsilon^{i j} u^{k}\right]+\frac{\rho}{e}\left[e \lambda_{2}\right]^{\star} g^{i j}\right)\right\} \equiv \kappa^{\Gamma} \sigma^{i j} .
\end{aligned}
$$

Equation (81) could be combined with Eq. (38) to give the canonical energy-momentum tensor; however we prefer to give the improved symmetric energy-momentum tensor given by Eq. (42) for independent metric, torsion, and nonmetricity since it is closer to previous work for spin fluids in Riemann-Cartan geometry. Substituting the Palatini tensor given by Eq. (43) into Eq. (42), we find our final results,

$$
\begin{aligned}
G^{(i j)} & +e^{-1} \stackrel{*}{\nabla} k\left[e\left(P^{i(j k)}+P^{j(k i)}-P^{k(i j)}\right)\right] \\
& =\kappa^{\Gamma} \sigma^{i j}+\frac{\kappa}{e} \stackrel{*}{\nabla}_{k}\left[e\left(-\rho \lambda_{2} u^{k} g^{i j}+u^{(i} S^{j) k}-\frac{1}{2} Y^{i j} u^{k}\right)\right]=\kappa\left(T_{T}^{i j}+T_{S}^{i j}+T_{T}^{i j}\right),
\end{aligned}
$$

where the perfect fluid energy-momentum tensor is given by

$$
T_{F}^{i j} \equiv[\rho(1+\epsilon)+p] u^{i} u^{j}+p g^{i j}
$$

the spin energy-momentum tensor is given by

$$
T_{S}^{i j} \equiv u^{(j} S^{i)} \dot{u}^{l}+u^{(j} S^{i) l} \dot{u}_{l}+\frac{1}{2 e} \stackrel{\nabla}{*}_{k}\left[e u^{(i} S^{j) k}\right]-\omega_{k}{ }^{i} S^{j) k}
$$

and the twist energy-momentum tensor is given by

$$
\begin{aligned}
T_{T}^{i j} & \equiv-u^{(j} \Upsilon^{i) l} \dot{u}_{l}+u^{(j} \Upsilon^{i)}{ }_{l} \dot{u}^{l}+2 v_{k}{ }^{(i} \Upsilon^{j) k}+v_{k}{ }^{(i} S^{j) k}-\frac{1}{2} v^{i j} \Upsilon_{l}{ }^{l} \\
& =-\dot{h}_{l}^{(j} \Upsilon^{i) l}+\dot{h}_{l}^{(j} \Upsilon^{i) l}+v_{k}{ }^{(i} S^{j) k}-\frac{1}{2} v^{i j} \Upsilon_{l}{ }^{l}
\end{aligned}
$$

The separation into spin and twist parts of the energy momentum is suggestive since both torsion and nonmetricity are present through the connection in $T_{S}^{i j}$ and since both spin and twist occur in $T_{T}^{i j}$; however the division is somewhat instructive since the Riemann-Cartan limit for the $T_{S}^{i j}$ has the same form, ${ }^{19}$ whereas $T_{T}^{i j}$ vanishes in the same limit.

In the next section, we calculate the variation for both particle number and volume conservation. 


\section{FLUID FIELD EQUATIONS FOR BOTH PARTICLE NUMBER AND VOLUME CONSERVATION}

\section{A. Lagrangian for spin-twist fluid: Particle number and volume conservation}

For the case of particle number and volume conservation, the Lagrangian will take the form

$$
{ }^{\dagger} \mathscr{L}={ }^{\dagger} \mathscr{C}_{\mathrm{REH}}+{ }^{\dagger} \mathscr{L}_{\mathrm{ST}}
$$

where the Ray-Einstein-Hilbert Lagrangian ${ }^{\dagger} \mathscr{W}_{\mathrm{REH}}$ is given by a geometric part

$$
i^{\dagger} \mathscr{B}_{G}=\frac{\dagger \mathscr{R}}{2 \kappa},
$$

where the dagger before a symbol represents the use of the volume conserving connection ${ }^{\dagger} \Gamma_{k j}{ }^{l}$ plus a perfect fluid Lagrangian given by

$$
{ }^{\dagger} \mathscr{L}_{F}=e\left\{-\rho\left[1+\epsilon\left(\rho, s, s_{i j}, \tau_{i j}\right)\right]+\lambda_{1}\left(u^{i} u^{j}+1\right)+\lambda_{2}^{\dagger} \nabla_{i}\left(\rho u^{i}\right)+\lambda_{3} u^{i} X_{, i}+\lambda_{4} u^{i} s_{, i}\right\},
$$

and the spin-twist Lagrangian is given by

$$
\begin{aligned}
\dagger \mathscr{L}_{\mathrm{ST}}= & e\left\{-\frac{1}{2} \rho k\left[a^{1 i} \dot{a}^{2}{ }_{i}-\dot{a}_{i}{ }_{i} a^{2 i}\right]-\frac{1}{2} \rho \tau a^{1 i} a^{2 j} u^{k \dagger} \nabla_{k} g_{i j}+\lambda_{11}\left(a^{1}{ }_{i} a^{1 i}-\eta^{11}\right)+\lambda_{12}\left(a^{1}{ }_{i} a^{2 i}-\eta^{12}\right)\right. \\
& \left.+\lambda_{22}\left(a^{2}{ }_{i} a^{2 i}-\eta^{22}\right)+\lambda_{41} u^{i} a^{1}{ }_{i}+\lambda_{42} u^{i} a^{2}{ }_{i}\right\} .
\end{aligned}
$$

Note that the "dot" derivative, i.e., the covariant derivative along fluid flow lines now takes the form $\dot{a}^{1 j} \equiv u^{k \dagger} \nabla_{k} a^{1 j}$. All other definition such as for the thermodynamics in Eq. (28) remain the same except for the obvious generalization using the volume conserving connection.

\section{B. Connection variational equations for case ${ }^{+*} \stackrel{\nabla}{k}_{k}\left[\rho u^{k}\right]=0$}

Using the constraint given by Eq. (22) will yield field equations in which the torsion vector is inactive. In addition we note that this constraint is independent of both torsion and nonmetricity. This means that this constraint will not contribute to the variation with respect to the connection ${ }^{\dagger} \Gamma_{k j}{ }^{l}$. Another way to see this is to rewrite the constraint in the form

$$
{ }^{\dagger} \stackrel{*}{\nabla}_{j}\left[\rho u^{j}\right] \equiv \stackrel{\{\}}{\nabla}_{j}\left[\rho u^{j}\right]=e^{-1} \partial_{j}\left[e \rho u^{j}\right]=0
$$

which shows that the $\delta^{\dagger} \Gamma_{k j}{ }^{l}$ variation of this term in the Lagrangian gives

$$
\delta_{+\Gamma}\left\{e \lambda_{2}{ }^{\dagger} \stackrel{\nabla}{\nabla}_{j}\left[\rho u^{j}\right]\right\}=\delta_{\dagger \Gamma}\left\{\lambda_{2} \partial_{j}\left[e \rho u^{j}\right]\right\}=0 \cdot \delta^{\dagger} \Gamma_{k j}^{l} .
$$

\section{Connection field equation}

Because of Eq. (91), the connection variation now becomes

Connection $\delta^{\dagger} \Gamma_{k j}^{l}$ :

$$
\frac{1}{2} u^{k} S_{l}^{j}+\frac{1}{2} u^{k} \Upsilon_{l}^{j}=-\frac{1}{\kappa}{ }^{\dagger} P_{l}^{j k}=-\Delta_{l}^{j k}
$$

In this case the first trace of the Palatini tensor gives

$$
{ }^{\dagger} P_{l}^{l k}=\frac{\kappa}{2} u^{k} \Upsilon_{x}^{x}=0
$$


or

$$
\lambda_{2}=-\tau \eta^{12}=0
$$

which implies that the anholonomic metric can be taken orthonormal. One should note that here the Palatini tensor is invariant under the particular torse transformation given by Eq. (17) which is called the volume preserving connection. ${ }^{22}$ Because of this, one finds ${ }^{\dagger} P_{l}^{j k}=P_{l}^{j k}$. Thus in what follows, we could drop the dagger on the Palatini tensors. We have instead opted to retain it so that the volume conserving connection is evident. Also it is now clear from the invariance of the Palatini tensor under the volume preserving transformation why the first trace vanishes; the Palatini tensor can only account for $60 \mathrm{deg}$ of freedom due to the connection variation. ${ }^{36}$ From the two other traces of the Palatini tensor, one gets

$$
{ }^{\dagger} P_{l j}^{l}=2^{\dagger} S_{j}+\frac{3}{2} \dagger Q_{x j}^{x}=0
$$

and

$$
{ }^{\dagger} P_{j l} l=-2^{\dagger} S_{j}+\frac{1}{2}{ }^{\dagger} Q^{x}{ }_{x j}=0 .
$$

This shows that the remaining traces can be resolved giving

$$
{ }^{\dagger} S_{j}={ }^{\dagger} Q^{x}{ }_{x j}=0 \text {. }
$$

Thus with all the traces vanishing, we find the torsion field equation

$$
{ }^{\dagger} \bar{S}_{l j}^{k}-\frac{1}{2}{ }^{\dagger} \overline{\bar{Q}}_{[l j]}^{k}=\frac{\kappa}{2} S_{l j} u^{k}
$$

and the nonmetricity field equation

$$
{ }^{\dagger} \overline{\bar{Q}}_{(l j)}{ }^{k}=\kappa \bar{Y}_{j l} u^{k}
$$

One should note the similarity between Eqs. (98) and Eqs. (52). Here we explicitly leave indication of the absence of traces to remind us that this is the case of volume conservation plus continuity constraint given by Eq. (22).

\section{Metric plus fluid field equations}

The remainder of the variational equations become

Metric equation $\delta g_{i j}$ :

$$
\begin{aligned}
& -\frac{G^{i j}}{2 \kappa}-\frac{1}{2} \rho(1+\epsilon) g^{i j}+\lambda_{1} u^{i} u^{j}+\lambda_{11} a^{1 i} a^{1 j}+\lambda_{22} a^{2 i} a^{2 j}+2 \lambda_{12} a^{(1 i} a^{2 j)}+2 \lambda_{41} u^{(i} a^{1 j)} \\
& \quad+2 \lambda_{42} u^{(i} a^{2 j)}-\frac{1}{2} T_{S} g^{i j}-\frac{1}{2} T_{T} g^{i j}+\frac{1}{2}{ }^{\dagger} \nabla_{k}\left[\rho k u^{k} a^{(1 j}\right] a^{2 i)}-\rho \dot{\lambda}_{2} g^{i j}-\frac{1}{2}^{\dagger} \nabla_{k}\left[\rho k u^{k} a^{(2 j}\right] a^{1 i)} \\
& \quad+\frac{1}{2}{ }^{\dagger} \nabla_{k}\left[\rho \tau u^{k} a^{(1 j j} a^{2 i)}\right]=0 .
\end{aligned}
$$

Fluid density $\delta \rho$ :

$$
-\left[(1+\epsilon)-\frac{p}{\rho}\right]-\dot{\lambda}_{2}-\frac{1}{\rho} T_{S}-\frac{1}{\rho} T_{T}=0 .
$$

Four velocity $\delta u^{k}$ : 


$$
\begin{aligned}
& -\rho \partial_{k} \lambda_{2}+2 \rho \lambda_{2} Q_{k}+2 \lambda_{2} u_{k}+\lambda_{3} \partial_{k} X+\lambda_{4} \partial_{k} s+2 \lambda_{41} a^{1}{ }_{k}+2 \lambda_{42} a^{2}{ }_{k}-\frac{1}{2} \rho k a^{1 i \dagger} \nabla_{k} a^{2}{ }_{i} \\
& \quad+\frac{1}{2} \rho k a^{2 i \dagger} \nabla_{k} a_{i}{ }_{i}-\frac{1}{2} \rho \tau\left[{ }^{\dagger} \nabla_{k} g_{i j}\right] a^{1 i} a^{2 j}=0 .
\end{aligned}
$$

Entropy \&s:

$$
\rho T-\stackrel{\{\}}{\nabla}_{i}\left[\lambda_{4} u^{i}\right]=0
$$

where the braces superscript signifies the Riemannian geometry covariant derivative.

Lin constraint $\delta \mathrm{X}$ :

$$
-\stackrel{\{\}}{i}_{i}\left[\lambda_{3} u^{i}\right]=0
$$

and the tetrad variations

$\delta a^{I k}$ :

$$
\begin{gathered}
-\rho k \omega_{k i} a^{2 i}-\rho \tau v_{i k} a^{2 i}+2 \lambda_{11} a_{k}^{1}+2 \lambda_{12} a_{k}^{2}+2 \lambda_{41} u_{k}-\frac{1}{2} \rho k \dot{a}_{k}^{2} \\
-\frac{1}{2} \dagger \nabla_{l}\left[\rho k a^{2 j} u^{l}\right] g_{j k}-\frac{1}{2} \rho \tau \dot{g}_{j k} a^{2 j}=0,
\end{gathered}
$$

and $\delta a^{2 k}$ :

$\rho k \omega_{k i} a^{1 i}-\rho \tau v_{i k} a^{1 i}+2 \lambda_{22} a^{2}{ }_{k}+2 \lambda_{12} a^{1}{ }_{k}+2 \lambda_{42} u_{k}-\frac{1}{2} \rho k \dot{a}^{1}{ }_{k}+\frac{1}{2} \dagger \nabla_{l}\left[\rho k a^{1 j} u^{l}\right] g_{j k}-\frac{1}{2} \rho \tau \dot{g}_{j k} a^{1 j}=0$.

The various constraint equations then completes the set of variational equations for the case with fluid continuity ${ }^{\ddagger} \stackrel{\nabla}{\nabla}_{i}\left[\rho u^{i}\right]=0$. Note that we explicitly left the continuity constraint in terms of the star derivative since it is the continuity constraint that makes the presence of the star derivative in the variational equations superfluous.

\section{E. Spin-twist transport equation}

It is interesting to note that the tetrad variational equations contain the possibility that the anholonomic metric is nondiagonal through the Lagrange multiplier $\lambda_{12}$. In this case we have explicitly assumed that the anholonomic metric was normalized. Because of the connection field equation, we see, a posteriori, that it can be taken orthonormal [see Eqs. (93)]. We find that the diagonal tetrad Lagrange multipliers take the form

$$
\lambda_{11}=\lambda_{22}=T_{S}+T_{T},
$$

whereas the nondiagonal component takes the form

$$
\lambda_{12}=\frac{1}{2} \rho \tau\left[\dot{a}_{k}{ }_{k} a^{1 k}+\dot{a}_{k}{ }_{k} a^{2 k}\right],
$$

and the spin and twist module functions are related by

$$
\dot{k}=2 \tau\left[\dot{a}_{k}{ }_{k} a^{1 k}-\dot{a}_{k}^{2} a^{2 k}\right] .
$$

Equation (64) now takes the form

$$
\rho \dot{\lambda_{2}}=[\rho(1+\epsilon)+p]+T_{S}+T_{T},
$$


whereas Eq. (65) for $\lambda_{1}$ remains the same as before. Also the symmetric and antisymmetric combinations of the Lagrange multipliers given by Equations (68) and (69), respectively, remain the same as before. However the antisymmetric combinations given by Eq. (74) changes slightly to

$$
\begin{gathered}
\rho k \omega_{i}{ }^{[k} s^{j] i}-2 \rho v_{i}{ }^{[k} \tau^{j] k}-u^{[j} S^{k]}{ }_{l} \dot{u}^{l}-u^{[j} S^{k] l} \dot{u}_{l}-u^{[k} Y^{j] l} \dot{u}_{l}+u^{[k} \Upsilon^{j]}{ }_{l} \dot{u}^{l}+\frac{1}{2} \rho k\left(\dot{a}_{l}{ }^{l[k} a^{2 j]}\right. \\
\left.-\dot{a}_{l} g^{l[k} a^{1 j]}\right)+\frac{1}{2} \rho k\left(\dot{a}^{[1 k} a^{2 j]}-\dot{a}^{[2 k} a^{1 j]}\right)+e^{-1} \rho(e k)^{\bullet} a^{[1 k} a^{2 j]}=0,
\end{gathered}
$$

so that the extended Fermi-Walker transport equation becomes

$$
\frac{1}{2} \rho \dot{s}^{k j}-v^{l[k} S^{j]}{ }_{l}+\omega_{l}{ }^{[k} S^{j] l}=u^{[k} S^{j] l} \dot{u}_{l}+u^{[k} S^{j l}{ }_{l} \dot{u}^{l}-\dot{h}_{l}^{[j} \Upsilon^{k] l}+\dot{h}^{l[j} \Upsilon^{k]}{ }_{l}
$$

Note again in RC, this reduces to the usual Fermi-Walker transport equation since in RC geometry

$$
\omega_{l}^{[k} S^{j] l} \equiv \frac{1}{2} \rho \dot{s}^{k j},
$$

which is broken in MA geometry because $\dot{k} \neq 0$ and therefore becomes

$$
\omega_{l}^{[k} S^{j] l}=\frac{1}{2} \rho \dot{s}^{k j}-v^{l[k} S^{j]}-\rho \dot{k} a^{[1 k} a^{2 j]} .
$$

Equation (112) can also be written equivalently in the form of Eq. (78) or (79) by replacing the * derivative by the dot derivative.

In the next section we develop the metric field equations.

\section{F. Metric field equations}

The symmetric combination of the tetrad equations given by Eq. (80) is the same for both fluid constraints. Therefore substituting Eqs. (65), (80), and (110) into Eq. (100) gives the metric field equation for independent metric and connection

$$
\begin{aligned}
G^{(i j)}= & \kappa\left\{\left([\rho(1+\epsilon)+p] u^{i} u^{j}+\rho g^{i j}\right)+u^{(j} S^{i)}{ }_{l} \dot{u}^{l}+u^{(j} S^{i) l} \dot{u}_{l}-u^{(j} \Upsilon^{i) l} \dot{u}_{l}+u^{(j} Y^{i)}{ }_{l} \dot{u}^{l}-\rho \omega_{k}{ }^{(i} S^{j) k}\right. \\
& \left.+2 \rho v_{k}{ }^{(i} \tau^{j) k}-v_{k}{ }^{(i} S^{j) k}+\frac{1}{2 k}{ }^{*}{ }^{*} \nabla_{k}\left[Y^{i j} u^{k}\right]\right\} \equiv \kappa^{\Gamma} \sigma^{i j},
\end{aligned}
$$

and then by Eq. (42), the improved symmetric energy momentum tensor becomes

$$
\kappa T^{i j} \equiv{ }^{\dagger} G^{(i j)}+{ }^{\dagger}{ }^{*}{ }_{k}\left({ }^{\dagger} P^{i(j k)}+{ }^{\dagger} P^{j(k i)}-{ }^{\dagger} P^{k(i j)}\right) \equiv \kappa\left(T_{F}^{i j}+T_{S}^{i j}+T_{T}^{i j}\right),
$$

where the perfect fluid energy-momentum tensor $T_{F}^{i j}$ is again given by Eq. (83), but the spin energy-momentum tensor is given by

$$
T_{S}^{i j} \equiv u^{(j} S^{i)} \dot{u}^{l}+u^{(j} S^{i) l} \dot{u}_{l}+\frac{1}{2} \stackrel{*}{\nabla}_{k}\left[u^{(i} S^{j) k}\right]-\omega_{k}^{(i} S^{j) k}
$$

and the twist energy-momentum tensor is given by

$$
T_{T}^{i j} \equiv-u^{(j} \Upsilon^{i) l} \dot{u}_{l}+u^{(j} \Upsilon^{i)}{ }_{l} \dot{u}^{l}+2 v_{k}{ }^{(i} \Upsilon^{j) k}+v_{k}{ }^{(i} S^{j) k}=-\dot{h}_{l}^{(j} \Upsilon^{i) l}+\dot{h}^{l(j} \Upsilon_{l}^{i)}+v_{k}{ }^{(i} S^{j) k}
$$

Note that due to Eq. (97), the star derivative in Eq. (117) is superfluous, but is left as a reminder of the fluid constraint. 


\section{DISCUSSIONS AND CONCLUSIONS}

In this work, we have described a straightforward generalization of a spin fluid in RC geometry to a fluid with both spin and twist in MA geometry. This generalization is based upon an extrapolation of the antisymmetric velocity gradient tensor which can only describe rigid body rotations. We have shown that in the generalization to both symmetric and antisymmetric components of the velocity gradient tensor (or stress tensor), one can describe both rotation and shear within a fluid. In order to describe a fluid with spin and twist in a space-time which is nearly flat (i.e., $R_{i j k}{ }^{l} \approx 0$ ), we have used the special relativistic Halbwachs' type description in an anholonomic frame which is then connected to the holonomic frame by the tetrads $a^{\alpha}{ }_{j}$ which in fact are the components of the transformation matrix between the two frames.

The direct application of the above formalism is to fluids in a metric affine space-time. In the case of spin, Brown ${ }^{37}$ has shown in a post-Newtonian approximation of a spin fluid in RC spacetime, that the components of the spin (intrinsic angular momentum) of the fluid contribute in the same level of approximation as ordinary angular momentum. Since the spin is directly related to the torsion through the torsion field equation, one can look on this as the geometrization of spin for applications in the astrophysical/gravitational setting. By contrast, one can devise a spin fluid purely within the general relativistic framework. ${ }^{20}$ However this framework is limited in the description of a spin fluid since no spin-squared terms contribute to the average of the symmetric energy momentum. ${ }^{38-40}$ The absence of spin-squared terms suggest that the correct way to include spin into the gravitational arena, is within a RC space-time. Thus since fluids can exhibit shear-like motions, i.e., twist, it seems reasonable to hypothesize the importance of MA space-time for these fluids. Indeed from the symmetry of the velocity gradient tensor, the symmetric component enter in the variational problem if and only if one considers the concept of nonmetricity.

Now look at the relationship between the frames in a different way. The word, physical, is understood through the above arguments to mean that the physical fluid resides in a MA geometry which we note is a curvilinear space-time. However this was only our tacit assumption (or identification) which makes that statement meaningful. That is, the tetrads $a^{\alpha}{ }_{j}$ are, as we have mentioned, just the components of the coordinate transformations between two different frames for which we chose to call one holonomic and the other anholonomic. The choice was ours.

Consider a different type of fluid such as the atmosphere on the surface of the earth. This fluid resides in a nearly flat spacetime. This means to lowest order in the graviatational field, we can treat the gravitational force in it's Newtonian form-constant near the surface of the earth. Thus if we assume that we can treat the frame of the Earth's atmosphere as a globally stationary Minkowskian frame, what we originally called the anholonomic frame is in fact the holonomic frame for this fluid. In this case, the constructs of spin and twist represent the geometrical expression of physical processes in the fluid such as vorticities subject to shear and expansion/ contraction. One then models these objects, and then spin-twist equations (84)-(85) or (117)(118) give the correct response of the fluid as a result of the conservation laws that are inherent in the variational approach, i.e., the application of the Bianchi identities for the graviational field tensor $G^{(i j)}{ }^{22}$ This will be the starting point for future work on specific examples of fluids.

\section{ACKNOWLEDGMENTS}

We are pleased to thank John R. Ray [Clemson] and Frank Madarasz [UAH] for interesting discussions and especially for directing our attention to further references for additional insight into the concept of twist.

\footnotetext{
${ }^{1}$ W. Israel, Nuovo Cimento 7, 860 (1973).

${ }^{2}$ W. Kopczyński, Phys. Lett. A 39, 219 (1972); 43, 63 (1973).

${ }^{3}$ I. Bailey and W. Israel, Commun. Math. Phys. 42, 65 (1975).

${ }^{4}$ I. Bailey, Ann. Phys. (NY) 119, 76 (1979).

${ }^{5}$ K. R. Symon, Mechanics (Addison-Wesley, Reading, MA, 1960), 2nd ed, Chap. 10.
} 
${ }^{6}$ W. Jaunzemis, Continuum Mechanics (Macmillan, New York, 1967). Note that in this text, the concept of rotation (or spin) in a continuum solid is referred to as twist, whereas what we call twist is referred to as extensions for deformable media.

${ }^{7}$ E. Kröner, Arch. Rat. Mech. Anal. 4, 273 (1960).

${ }^{8}$ E. Kröner, in Volesungen über theoretische Physik, edited by A. Sommerfeld (Verlagsges., Leipzig, 1964), 5th ed., Chap. 9.

${ }^{9}$ See also additional references in F. W. Hehl, G. D. Kerlick, and P. von der Heyde, Z. Naturforsch 31a, 111 (1976).

${ }^{10}$ G. A. Maugin and A. C. Eringer, J. Math. Phys. 13, 143, 1777, 1788 (1972).

"F. W. Hehl, G. D. Kerlick, and P. von der Heyde, Z. Naturforsch. 31a, 111, 524, 823 (1976).

${ }^{12}$ L. L. Smalley, Phys. Lett. A 61, 436 (1977).

${ }^{13}$ F. W. Hehl, E. A. Lord, and G. D. Kerlick, Gen. Rel. Grav. 9, 691 (1978).

${ }^{14}$ F. W. Hehl, E. A. Lord, and Y. Ne'eman, Phys. Lett. B 71, 432 (1977); Phys. Rev. D. 17, 428 (1978).

${ }^{15}$ V. N. Ponomariev and Y. N. Obukhov, Gen. Rel. Grav. 14, 309 (1982).

${ }^{16}$ E. A. Lord, Phys. Lett. A 65, 1 (1978).

${ }^{17}$ Y. N. Obukhov and R. Tresguerres, Phys. Lett. A 184, 17 (1993).

18 J. R. Ray, J. Math. Phys. 13, 1451 (1972).

${ }^{19}$ J. R. Ray and L. L. Smalley, Phys. Rev. Lett. 49, 1059 (1982); 50, 626E (1983).

${ }^{20}$ J. R. Ray, L. L. Smalley, and J. P. Krisch, Phys. Rev. D 35, 3261 (1987).

${ }^{21}$ T. Halbwachs, Theorie relativiste des fluid à spin (Gautièr-Villars, Paris, 1960).

${ }^{22}$ J. A. Schouten, Ricci Calculus (Springer-Verlag, Berlin, 1954), 2nd ed.

${ }^{23}$ F. W. Hehl. Gen. Rel. Grav, 4, 333 (1973); 5, 491 (1974).

${ }^{24}$ L. L. Smalley and J. R. Ray, Phys. Lett. A 134, 87 (1988).

25 J. Frenkel, Z. Phys. 37, 243 (1926).

${ }^{26}$ F. W. Hehl, G. D. Kerlick, E. A. Lord, and L. L. Smalley, Phys. Lett. B 70, 70 (1977).

${ }^{27}$ F. W. Hehl and G. D. Kerlick, Gen. Rel. Grav. 9, 691 (1978).

${ }^{28}$ F. W. Hehl, E. A. Lord, and L. L. Smalley, Gen. Rel. Grav. 13, 1037 (1981).

${ }^{29}$ L. L. Smalley, Gen. Rel. Grav. 10, 1179 (1993).

${ }^{30} \mathrm{R}$. R. Wahba, "Parametrization of cosmological scale factor during inflationary times," Dissertation, University of Alabama in Huntsville (1989).

${ }^{31}$ L. L. Smalley, Class. Quantum Grav. 10, 1179 (1993).

${ }^{32}$ See, for example, the work on elasticity by R. L. Seliger and G. B. Whitham, Proc. R. Soc. London, Ser. A 305, 1 (1968).

${ }^{33}$ L. L. Smalley, Phys. Lett. A 61, 436 (1977).

${ }^{34}$ F. W. Hehl, P. von der Heyde, G. D. Kerlick, and J. M. Nester, Rev. Mod. Phys. 48, 393 (1976).

${ }^{35}$ See, for example, J, D. McCrea. Class. Quantum Grav. 9, 553 (1992).

${ }^{36}$ For an explanation of this effect, see F. W. Hehl and G. D. Kerlick, Gen. Rel. Grav. 9, 691 (1978)

${ }^{37} \mathrm{~J}$. P. Brown, "The post-Newtonian approximation for self-consistent perfect fluids with spin density," thesis, University of Alabama in Huntsville, (1989).

${ }^{38}$ L. L. Smalley and J. P. Krisch, Class. Quantum Grav. 11, 2517 (1994).

${ }^{39}$ M. A. P. Martin, E. P. Vasioncellos-Vaidya, and M. M. Som, Class. Quantum Grav. 8, 2225 (1991).

${ }^{40}$ L. L. Smalley and J. P. Krisch, "Spinning Fluid Cosmology in Einstein-Cartan Theory," Class. Quantum Grav., in press, 1994. 